\title{
Anemia e deficiência de vitamina A em crianças menores de cinco anos assistidas pela Estratégia Saúde da Família no Estado de Pernambuco, Brasil
}

\author{
Anemia and vitamin A deficiency in children under five years old \\ attended under the Family Health Program \\ in the State of Pernambuco, Brazil
}

Weslla Karla Albuquerque Silva de Paula ${ }^{1}$

Maria de Fátima Costa Caminha ${ }^{1}$

José Natal Figueirôa ${ }^{1}$

Malaquias Batista Filho ${ }^{1}$

\footnotetext{
${ }^{1}$ Instituto de Medicina Integral Prof. Fernando Figueira. R. Coelhos 300 Boa Vista. 50.070-550 Recife PE Brasil. weslla19@hotmail.com
}

\begin{abstract}
The scope of this study was to describe the prevalence and factors associated with anemia and vitamin A deficiency (VAD) in children under five years old assisted under the Family Health Program. A cross-sectional study was conducted in Pernambuco, Brazil, in 2006. Children between 6 and 59 months-old, who had performed measurements of hemoglobin and serum retinol, were selected using samples from 945 and 563 children respectively. Anemia was determined by the hemoglobin level $<11 \mathrm{~g} / \mathrm{dL}$ and VAD by serum retinol $<\mu \mathrm{mol} / \mathrm{L}$. Univariate and multivariate analyses were performed by Poisson multiple regression using a hierarchical model. Anemia prevalence was 35\%, decreasing with the increase number of people per room, maternal age and the child's age. With respect to VAD, its prevalence was $16 \%$, rising in places where garbage disposal was inadequate and in children who had suffered from diarrhea in the previous fifteen days. The prevalence of anemia was more than double that found for VAD, highlighting the influence of environmental factors on VAD.
\end{abstract}

Key words Anemia, Vitamin A deficiency, Epidemiology, Family health program, Nutritional surveys
Resumo O objetivo do presente estudo foi descrever a prevalência e os fatores associados à anemia e à deficiência de vitamina A (DVA) em crianças menores de cinco anos assistidas pela Estratégia Saúde da Família. Estudo transversal, realizado em Pernambuco, Brasil, em 2006. Foram selecionadas crianças entre 6 e 59 meses de idade, que tiveram realizadas dosagens de hemoglobina e retinol sérico, perfazendo amostras de 945 e 563 crianças respectivamente. A presença de anemia foi determinada pelo nível de hemoglobina $<11 \mathrm{~g} / \mathrm{dL}$ e DVA pelo nível de retinol sérico $<0,70 \mu \mathrm{mol} / \mathrm{L}$. Realizaram-se análises univariada e ajustada por regressão múltipla de Poisson, utilizando modelo hierarquizado. A prevalência de anemia foi de $35 \%$, diminuindo com o aumento do número de pessoas por cômodo, idade materna e idade da criança. No que concerne à DVA, sua prevalência foi de $16 \%$, aumentando em locais onde o destino do lixo era inadequado e em crianças que apresentaram diarreia nos últimos quinze dias. A prevalência de anemia foi maior que o dobro encontrada para a DVA, chamando atenção a influência dos fatores ambientais sobre a DVA.

Palavras-chave Anemia, Deficiência de vitamina A, Epidemiologia, Programa saúde da família, Inquéritos nutricionais 


\section{Introdução}

No rol das doenças carenciais específicas, a anemia ferropriva e a deficiência de vitamina A (DVA) configuram-se como importantes problemas de saúde pública, em virtude de suas elevadas prevalências e consequências adversas, especialmente em países em desenvolvimento e entre grupos mais vulneráveis, como crianças menores de cinco anos e mulheres em idade fértil ${ }^{1,2}$.

A anemia ocorre, predominantemente, nas situações onde a deficiência de ferro se torna prolongada, estando associada à prematuridade e baixo peso ao nascer e maior probabilidade de déficits no desenvolvimento motor, cognitivo e comprometimento do sistema imunológico da criança ${ }^{3}$. Com distribuição mundial, esta deficiência nutricional também prevalece em países desenvolvidos, ainda que em menores proporções. A Organização Mundial de Saúde (OMS), em 2009, publicou um relatório global sobre anemia, estimando prevalência mundial na ordem de $24,8 \%$, ou seja, acometendo 1,62 bilhão de pessoas, especialmente crianças, com frequência de $47,4 \%{ }^{4}$. No Brasil, segundo a Pesquisa Nacional de Demografia e Saúde da Criança e da Mulher (PNDS-2006) ${ }^{5}$, a prevalência de anemia, em crianças menores de cinco anos é de $21 \%$, variando consideravelmente entre as regiões.

$\mathrm{Na}$ segunda representação epidemiológica, encontra-se a deficiência de vitamina A (DVA). A vitamina A é um importante micronutriente, por seu efeito protetor na prevenção de estruturas e funções oculares, integridade cutânea, sistema imunológico e redução da morbimortalidade por doenças infecciosas, como em relação às complicações da diarreia e infecções respiratórias agudas (IRA) ${ }^{6}$. Segundo a OMS, estima-se que 190 milhões de crianças menores de 5 anos possuam baixa concentração de retinol sérico $(<0,70 \mu \mathrm{mol} /$ L), comprometendo $33,3 \%$ da população de préescolares em escala mundial ${ }^{2}$. No Brasil, a DVA apresenta prevalência de $17,4 \%$ entre crianças de 6 a 59 meses de idade, predominando nas regiões Nordeste $(19 \%)$ e Sudeste $(21,6 \%)^{5,7}$. Segundo Ramalho et al. ${ }^{8}$, as piores situações são encontradas nos estados de São Paulo, Minas Gerais, Pernambuco, Paraíba e Amazonas.

Diante deste cenário desfavorável, há algum tempo o Ministério da Saúde (MS) desenvolve estratégias visando o enfrentamento destes e outros problemas nutricionais, a exemplo da Política Nacional de Alimentação e Nutrição $(\text { PNAN })^{9}$; a obrigatoriedade da fortificação das farinhas de milho e trigo com ferro e ácido fólico, a partir de $2004^{10}$; o Programa Nacional de Suplementação de Vitamina $\mathrm{A}^{11}$ e do Programa Nacional de Suplementação de Ferro, em 2005, com suplementação medicamentosa preventiva de ferro a crianças de 6 a 18 meses de idade e a mulheres grávidas, nas unidades básicas de saúde do Sistema Único de Saúde (SUS) ${ }^{12}$.

Decorridos pouco mais de 10 anos da PNAN, ainda são colocados desafios e propostas para o fortalecimento das ações de alimentação e nutrição no âmbito do SUS, em especial na atenção básica à saúde $(\mathrm{ABS})^{13}$. Desta maneira, iniciativas de promoção de práticas alimentares e estilo de vida saudáveis, programas para prevenção e controle das alterações nutricionais, monitoramento da situação alimentar e nutricional, controle social direcionado à nutrição no SUS, a intersetorialidade, dentre outras propostas, foram reiteradas pelo MS, como ações importantes para serem desenvolvidas na ABS, em especial pela Estratégia Saúde da Família (ESF) ${ }^{13}$.

Concebida em 1994, ainda como programa, a ESF constitui um modelo de reorientação da atenção básica, pautada nos princípios do SUS, que desenvolve ações de promoção à saúde, prevenção e recuperação de agravos, tendo a família como centro do cuidado ${ }^{14,15}$, devendo, dentre outras ações, realizar cadastramento domiciliar, diagnóstico situacional, desenvolver ações dirigidas aos problemas de saúde de maneira pactuada com a comunidade e cuidar dos indivíduos e famílias, de forma a manter postura pró-ativa frente aos problemas de saúde-doença da população ${ }^{16}$. A ESF oferece cobertura a mais da metade das famílias brasileiras $(52,6 \%$, totalizando mais de 100 milhões de pessoas), e em Pernambuco estima-se quase $70 \%$ de cobertura populacional ${ }^{17}$.

Entretanto, são escassas as informações disponíveis sobre anemia e DVA nas populações infantil e de pré-escolares, no âmbito mais amplo da população assistida pela ESF. Neste sentido, considerando a importância dos problemas nutricionais e a consolidação das políticas públicas de alimentação e nutrição na esfera do SUS, os objetivos da estratégia e a cobertura da população por ela beneficiada, bem como o potencial de ações que podem ser desenvolvidas, torna-se oportuno, pertinente e relevante, descrever a prevalência e os fatores associados à anemia e deficiência de vitamina A em crianças menores de cinco anos assistidas pela ESF no Estado de Pernambuco. 


\section{Métodos}

Trata-se de estudo transversal, com componentes analíticos, utilizando dados secundários do banco de informações da III Pesquisa Estadual de Saúde e Nutrição (PESN), inquérito populacional realizado no estado de Pernambuco, entre os meses de maio a outubro de 2006, com o objetivo geral de atualizar e ampliar o diagnóstico da situação de saúde, nutrição, alimentação e condições socioeconômicas da população do Estado, com ênfase no grupo materno-infantil ${ }^{18}$.

A amostragem da III PESN foi do tipo probabilística, escolhida em múltiplos estágios pelo sorteio sequencial de municípios, setores censitários e domicílios, sendo dimensionada para assegurar representatividade estatística de dois estratos geoeconômicos: Urbano e Rural. Fizeram parte da amostra 18 municípios indicados por sorteio aleatório, com probabilidade quase proporcional à sua população, de modo que os de maiores efetivos demográficos tiveram maiores chances de serem sorteados. Foram excluídas da amostra, crianças com malformações congênitas e doenças graves que afetassem significantemente o crescimento ou impedissem sua avaliação.

Para o trabalho de campo deste inquérito populacional em Pernambuco contou-se com 15 pesquisadores distribuídos em cinco equipes (coordenação de área, supervisão de campo, entrevistadores, laboratório e antropometria), treinados previamente, com vistas às padronizações dos procedimentos e preenchimento de coleta dos dados. As entrevistas eram realizadas com a pessoa responsável pela criança. Na impossibilidade de realizar a entrevista, após duas tentativas, com a pessoa previamente determinada, por motivo de ausência ou de mudança de endereço, o supervisor de campo procedia a sua substituição nas casas de números seguintes, desde que houvesse uma criança com a mesma faixa etária nesta outra família.

A dosagem de hemoglobina foi realizada em crianças entre 6 meses e 5 anos, em amostra de sangue venoso, por meio do equipamento HEMOCUE, de leitura imediata, mediante punção venosa, estabelecendo-se o diagnóstico de anemia com base no critério recomendado pela OMS, considerando anêmicas as crianças com hemoglobina abaixo de $11 \mathrm{~g} / \mathrm{dL}^{1}$. A determinação do retinol sérico foi realizada no Laboratório no Centro de Investigação em Micronutrientes CIMICRON, do Hospital Universitário Lauro Wanderley da Universidade Federal da Paraíba, usando o método de cromatografia liquida de alta pressão - HPLC (High Pressure Liquid Chromatography), segundo a técnica estabelecida por Furr et al. ${ }^{19}$, considerando-se o ponto de corte $<$ $0,70 \mu \mathrm{mol} / \mathrm{L}$ para caracterizar baixo nível de retinol sérico, consoante classificação da $\mathrm{OMS}^{2}$.

Do total de 1650 crianças menores de 5 anos analisadas na III PESN/PE-2006 ${ }^{18}, 1105$ eram assistidas pela ESF compondo um arquivo "ad hoc" com as demais variáveis de interesse. Contudo, para atender aos objetivos do presente estudo, foram selecionadas apenas as crianças entre 6 e 59 meses de idade, de ambos os sexos, que tiveram realizadas as dosagens de hemoglobina e retinol sérico.

Para o cálculo do tamanho amostral deste estudo, utilizou-se o "StatCalc" do software Epiinfo, versão 3.5.1. Tendo em vista a análise de dois desfechos, foram calculadas amostras individuais a partir de diferentes prevalências dos eventos (16\% para DVA e $34 \%$ para anemia $\left.{ }^{18}\right)$, como base populacional a estimativa de 283.075 crianças menores de cinco anos cadastradas nas ESF do Estado de Pernambuco ${ }^{20}$, margem de erro de $\pm 3 \%$ e intervalo de confiança de $95 \%$. Assim calculadas, seriam necessárias 572 e 955 crianças para responderem as questões do estudo referentes às ocorrências de DVA e anemia, respectivamente. No final, as amostras reais, pela ordem de descrição, foram respectivamente de 563 e 945 crianças menores de cinco anos.

As prevalências dos desfechos (anemia e DVA) foram analisadas segundo associação com variáveis preditoras (socioeconômicas, demográficas, ambientais, maternas, acesso aos serviços de saúde e relacionadas à criança). Utilizando-se regressão de Poisson simples, com a escolha da variância robusta para as estimativas dos parâmetros, realizou-se análise bivariada para se obter as estimativas pontuais e intervalares das razões de prevalências e testar as associações das variáveis explanatórias com os desfechos. Para as estimativas intervalares, adotou-se o nível de confiança de 95\%. As significâncias estatísticas das associações foram avaliadas com o teste da razão de verossimilhança, sendo considerado estatisticamente significante $\mathrm{p}<0,05$.

A estratégia delineada para a análise multivariada seguiu um modelo hierarquizado para a determinação da DVA e anemia, previamente proposto, hipoteticamente baseado nas relações lógicas e teóricas entre os eventos e seus fatores determinantes ${ }^{21-24}$. Nesta concepção, as variáveis foram agrupadas em três blocos (Figura 1). No primeiro, foram relacionados fatores socioeconômicos e ambientais. No segundo, foram in- 


\section{Bloco I}

Fatores socioeconômicos

. Renda familiar

- Bens de consumo

. Escolaridade materna

- Trabalho materno
Fatores ambientais

. Estrato geográfico

. Tipo de habitação

- Abastecimento de água

- Tratamento de água

de beber

. Presença de

banheiro

- Destino dos dejetos

- Destino do lixo

\begin{tabular}{|c|c|}
\hline \multicolumn{2}{|c|}{ Bloco II } \\
\hline Fatores maternos & \multirow{2}{*}{$\begin{array}{c}\text { Fatores relacionados } \\
\text { ao acesso aos serviços } \\
\text { de saúde }\end{array}$} \\
\hline $\begin{array}{l}\text { Idade materna } \\
\text { Realizacão de }\end{array}$ & \\
\hline $\begin{array}{l}\text { ré-natal } \\
\text { Node filhos }<5 \text { anos }\end{array}$ & $\begin{array}{l}\text {. Distância do serviço de } \\
\text { saúde } \\
\text {. Recebe visita do ACS }\end{array}$ \\
\hline
\end{tabular}

\section{Bloco III}

Fatores relacionados à criança

\begin{tabular}{l}
\hline . Idade \\
. Sexo \\
. Peso ao nascer \\
. Suplementação de vitamina A \\
. Ocorrência de morbidades \\
(diarreia, tosse e febre) \\
. Ocorrência de hospitalização
\end{tabular}

\section{ANEMIA}

DVA

Figura 1. Modelo causal hierarquizado do processo de determinação de anemia e déficit de vitamina A em crianças menores de cinco anos, no estado de Pernambuco (2006).

cluídas variáveis referentes a fatores maternos e de acesso ao serviço de saúde. Por fim, posicionados no nível mais proximal do modelo, o terceiro bloco foi constituído por variáveis relacionadas à criança.

Para se obter as estimativas ajustadas das razões de prevalência, utilizou-se regressão múltipla de Poisson, sendo realizada análise multivariada, seguindo a estratégia proposta por Victora et al. ${ }^{25}$. De acordo com essa estratégia, as variá- veis dos níveis hierárquicos definidos a priori foram incluídas no modelo, nível por nível.

Em cada nível hierárquico, a seleção de variáveis para o modelo foi realizada com o procedimento "backwards", utilizando-se como critério de exclusão, em cada passo, o valor $\mathrm{p} \leq 0,20$. Ao término do processo em um bloco, cada variável excluída era novamente reintroduzida e reavaliada sua significância estatística. No modelo final, foram consideradas significantes aquelas variáveis de cada bloco que apresentaram um valor $\mathrm{p}$ $<0,05$. As análises estatísticas foram realizadas com o software Stata 12.1SE.

A pesquisa foi conduzida segundo os padrões requeridos pela Resolução 196/96 ${ }^{26}$ e aprovado pelo Comitê de Ética em Pesquisa (CEP) do IMIP.

\section{Resultados}

Entre as crianças menores de cinco anos assistidas pelas ESF no Estado de Pernambuco, as prevalências de DVA e anemia foram, aproximadamente, $16 \%$ e $35 \%$, respectivamente.

As Tabelas 1 e 2 mostram os resultados das análises brutas e ajustadas para os dois desfechos estudados. Em relação à DVA, dos 23 subgrupos de variáveis categorizadas para a análise preliminar de associações, ou seja, razões brutas de prevalência, no bloco I, apenas a renda familiar per capita $<0,25$ SM e nenhuma escolaridade materna, as condições de saneamento (abastecimento de água, presença de banheiro e destino do lixo sem coleta pública) alcançaram o limiar adotado $(0,20)$ para ingressarem na etapa seguinte do modelo de análise. No bloco III, reunindo as variáveis referentes às crianças (fatores proximais), o baixo peso ao nascer e a ocorrência de diarreia alcançaram valores de $\mathrm{p}$ abaixo do nível seletivo adotado para as análises bivariadas. Por fim, ao se fazer as análises ajustadas intra e intergrupos, somente o destino do lixo e ocorrência de diarreia nos últimos 15 dias foram mantidos no modelo explicativo final (Tabela 1).

No que concerne à anemia, todas as variáveis agrupadas como fatores socioeconômicos (renda familiar, bens de consumo, escolaridade materna e trabalho da mãe) alcançaram os níveis mínimos $(0,20)$ convencionados para o "screening" estatístico das análises bivariadas, ou seja, da comparação de prevalências brutas. $\mathrm{O}$ tratamento de água de beber, a ausência de banheiro, o destino inadequado dos dejetos e o número de pessoas por cômodo foram demarcados de diferenças significativas. Ainda em referência ao blo- 
Tabela 1. Associação entre fatores socioeconômicos, demográficos, ambientais, biológicos e DVA em crianças menores de cinco anos assistidas pelas ESF. Pernambuco, 2006.

\begin{tabular}{|c|c|c|c|c|c|c|}
\hline Blocos/Variáveis & $\begin{array}{c}\text { Amostra } \\
(\mathrm{n}=563) \\
n^{\mathrm{a}}\end{array}$ & DVA\% & $\begin{array}{c}\mathrm{RP}_{\text {bruta }} \\
(\mathrm{IC} \text { 95\%) }\end{array}$ & p & $\begin{array}{l}\mathrm{RP}_{\text {ajustada }} \\
\text { (IC 95\%) }\end{array}$ & $\mathbf{p}$ \\
\hline \multicolumn{7}{|l|}{ Bloco I } \\
\hline \multicolumn{7}{|l|}{ Fatores socioeconômicos } \\
\hline Renda familiar per capita & & & & 0,075 & & $0,229^{*}$ \\
\hline$<0,25 \mathrm{SM}$ & 330 & $62(18,8)$ & $1,6(0,8-3,1)$ & & $1,3(0,7-2,7)$ & \\
\hline $0,25-0,5 \mathrm{SM}$ & 158 & $20(12,7)$ & $1,1(0,5-2,3)$ & & $1,0(0,5-2,2)$ & \\
\hline$\geq 0,50 \mathrm{SM}$ & 67 & $8(11,9)$ & 1,0 & & 1,0 & \\
\hline Bens de consumo & & & & 0,343 & & \\
\hline 0-2 bens & 262 & $46(17,6)$ & $1,2(0,8-1,7)$ & & & \\
\hline 3-5 bens & 301 & $44(14,6)$ & 1,0 & & & \\
\hline Escolaridade materna & & & & $0,187^{\star}$ & & $0,614^{*}$ \\
\hline Nenhuma & 53 & $12(22,6)$ & $1,7(0,8-3,4)$ & & $1,2(0,5-2,8)$ & \\
\hline $1-8$ anos & 409 & $64(15,6)$ & $1,3(0,7-2,0)$ & & $0,9(0,5-1,7)$ & \\
\hline$\geq 9$ anos & 97 & $13(13,4)$ & 1,0 & & 1,0 & \\
\hline Trabalho materno & & & & 0,248 & & \\
\hline Sim & 140 & $18(12,9)$ & 1,0 & & & \\
\hline Não & 422 & $72(17,1)$ & $1,3(0,8-2,1)$ & & & \\
\hline \multicolumn{7}{|l|}{ Fatores ambientais } \\
\hline Estrato geográfico & & & & 0,257 & & \\
\hline RMR & 159 & $19(11,9)$ & 1,0 & & & \\
\hline IU & 98 & $16(16,3)$ & $1,4(0,7-2,5)$ & & & \\
\hline IR & 306 & $55(18,0)$ & $1,5(0,9-2,4)$ & & & \\
\hline Tipo de habitação & & & & 0,981 & & \\
\hline Alvenaria/Tijolo & 544 & $87(16,0)$ & 1,0 & & & \\
\hline Outros & 19 & $3(15,8)$ & $1(0,3-2,8)$ & & & \\
\hline Abastecimento de água & & & & 0,17 & & 0,219 \\
\hline Rede geral & 269 & $37(13,8)$ & 1,0 & & 1,0 & \\
\hline Outros & 294 & $53(18,0)$ & $1,3(0,9-1,9)$ & & $1,4(0,8-2,4)$ & \\
\hline Tratamento de água de beber & & & & 0,287 & & \\
\hline Adequado & 273 & $39(14,3)$ & 1,0 & & & \\
\hline Não adequado & 290 & $51(17,6)$ & $1,2(0,8-1,8)$ & & & \\
\hline Presença de banheiro & & & & 0,082 & & 0,474 \\
\hline Sim & 405 & $58(14,3)$ & 1,0 & & 1,0 & \\
\hline Não & 158 & $32(20,3)$ & $1,4(1-2,1)$ & & $1,2(0,7-1,9)$ & \\
\hline Destino dos dejetos & & & & 0,604 & & \\
\hline Rede geral & 163 & $24(14,7)$ & 1,0 & & & \\
\hline Outros & 400 & $66(16,5)$ & $1,1(0,7-1,7)$ & & & \\
\hline Destino do lixo & & & & 0,021 & & 0,021 \\
\hline Coleta pública & 301 & $38(12,6)$ & 1,0 & & 1,0 & \\
\hline Outros & 262 & $52(19,8)$ & $1,6(1,1-2,3)$ & & $1,6(1,1-2,3)$ & \\
\hline No de pessoas por cômodo & & & & $0,353^{*}$ & & \\
\hline$<1$ & 231 & $43(18,6)$ & 1,0 & & & \\
\hline $1-2$ & 302 & $43(14,2)$ & $0,8(0,5-1,1)$ & & & \\
\hline$\geq 2$ & 30 & $4(13,3)$ & $0,7(0,3-1,9)$ & & & \\
\hline
\end{tabular}

co I, também se enquadraram no critério de seleção as variáveis idade materna e número de filhos. Por fim, na análise bivariada dos fatores referentes às crianças (bloco III) foram incluídas a idade da criança ( $<2$ anos) e a ocorrência de diarreia como fatores de diferenciação estatística de prevalência. Já na etapa conclusiva do modelo (análise multivariada), mantiveram-se apenas 
Tabela 1. continuação

\begin{tabular}{|c|c|c|c|c|c|c|}
\hline Blocos/Variáveis & $\begin{array}{c}\text { Amostra } \\
(n=563) \\
n^{a}\end{array}$ & DVA\% & $\begin{array}{c}\mathbf{R P}_{\text {bruta }} \\
\text { (IC 95\%) }\end{array}$ & $\mathbf{p}$ & $\begin{array}{l}\mathrm{RP}_{\text {ajustada }} \\
\text { (IC 95\%) }\end{array}$ & $\mathbf{p}$ \\
\hline \multicolumn{7}{|l|}{ Bloco II } \\
\hline \multicolumn{7}{|l|}{ Fatores maternos } \\
\hline Idade materna & & & & $0,684^{*}$ & & \\
\hline$<20$ anos & 53 & $9(17,0)$ & $1,1(0,6-2,2)$ & & & \\
\hline $20 \mathrm{a}-30 \mathrm{a}$ & 286 & $47(16,4)$ & $1,1(0,7-1,6)$ & & & \\
\hline$\geq 30 \mathrm{a}$ & 223 & $34(15,2)$ & 1,0 & & & \\
\hline Realização de pré-natal & & & & 0,256 & & \\
\hline Nenhuma & 9 & $3(33,3)$ & $2,1(0,8-5,4)$ & & & \\
\hline $1-5$ consultas & 127 & $18(14,2)$ & $0,9(0,5-1,4)$ & & & \\
\hline 6 ou mais & 393 & $63(16,0)$ & 1,0 & & & \\
\hline No de filhos $<5$ anos & & & & 0,817 & & \\
\hline 1 filho & 323 & $49(15,2)$ & 1,0 & & & \\
\hline 2 filhos & 185 & $32(17,3)$ & $1,1(0,8-1,7)$ & & & \\
\hline 3 ou mais filhos & 55 & $9(16,4)$ & $1,1(0,6-2,1)$ & & & \\
\hline \multicolumn{7}{|l|}{ Acesso serviços de saúde } \\
\hline Distância serviços de saúde & & & & 0,437 & & \\
\hline $0-10 \mathrm{~min}$ & 155 & $27(17,4)$ & 1,0 & & & \\
\hline $10-30 \mathrm{~min}$ & 243 & $32(13,2)$ & $0,8(0,5-1,2)$ & & & \\
\hline$\geq 30 \mathrm{~min}$ & 160 & $27(16,9)$ & $1(0,6-1,6)$ & & & \\
\hline Recebe visita ACS & & & & 0,779 & & \\
\hline Sim & 499 & $79(15,8)$ & 1,0 & & & \\
\hline Não & 64 & $11(17,2)$ & $1,1(0,6-1,9)$ & & & \\
\hline \multicolumn{7}{|l|}{ Bloco III } \\
\hline \multicolumn{7}{|l|}{ Fatores relacionados à criança } \\
\hline Idade & & & & 0,634 & & \\
\hline$<2$ anos & 188 & $32(17,0)$ & $1,1(0,7-1,6)$ & & & \\
\hline$\geq 2$ anos & 375 & $58(15,5)$ & 1,0 & & & \\
\hline Sexo & & & & 0,726 & & \\
\hline Masculino & 297 & $49(16,5)$ & $1,1(0,7-1,6)$ & & & \\
\hline Feminino & 266 & $41(15,4)$ & 1,0 & & & \\
\hline Peso ao nascer & & & & $<0,001$ & & 0,201 \\
\hline$<2.500 \mathrm{~g}$ & 34 & $8(23,5)$ & $3,7(2,3-5,9)$ & & $1,5(0,8-2,8)$ & \\
\hline$>2.500 \mathrm{~g}$ & 514 & $79(15,4)$ & 1,0 & & 1,0 & \\
\hline Suplementação vit. A & & & & 0,358 & & \\
\hline Sim & 281 & $49(17,4)$ & 1,0 & & & \\
\hline Não & 281 & $41(14,6)$ & $0,8(0,6-1,2)$ & & & \\
\hline Ocorrência diarreia ${ }^{*}$ & & & & 0,036 & & 0,035 \\
\hline Sim & 101 & $23(22,8)$ & $1,6(1-2,4)$ & & $1,6(1,0-2,4)$ & \\
\hline Não & 462 & $67(14,5)$ & 1,0 & & 1,0 & \\
\hline Ocorrência tosse e febre ${ }^{*}$ & & & & 0,342 & & \\
\hline Sim & 105 & $20(19,0)$ & $1,2(0,8-1,9)$ & & & \\
\hline Não & 457 & $70(15,3)$ & 1,0 & & & \\
\hline Internação hospitalar & & & & 0,21 & & \\
\hline Sim & 102 & $12(11,8)$ & $0,7(0,4-1,2)$ & & & \\
\hline Não & 461 & $78(16,9)$ & 1,0 & & & \\
\hline
\end{tabular}

${ }^{\mathrm{a}}$ Os totais parciais de cada grupo podem diferir do total da amostra em virtude de informações ignoradas pelas entrevistadas; * Utilizado teste de Wald de tendência linear; ${ }^{\dagger}$ Salário Mínimo (SM) vigente no ano de 2006 (R \$350,00); ${ }^{*}$ Considerou-se ocorrência de diarreia nas duas últimas semanas anteriores à entrevista para a coleta dos dados, a ocorrência de tosse e febre na semana anterior e internação hospitalar da criança nos últimos doze meses anteriores à entrevista para a coleta dos dados. 
Tabela 2. Associação entre fatores socioeconômicos, demográficos, ambientais, biológicos e anemia em crianças menores de cinco anos assistidas pelas ESF. Pernambuco, 2006.

\begin{tabular}{|c|c|c|c|c|c|c|}
\hline Blocos/Variáveis & $\begin{array}{c}\text { Amostra } \\
(\mathrm{n}=945) \\
\mathrm{n}^{\mathrm{a}}\end{array}$ & $\begin{array}{c}\text { Anemia } \\
\%\end{array}$ & $\begin{array}{c}\mathbf{R P}_{\text {bruta }} \\
\text { (IC 95\%) }\end{array}$ & $\mathbf{p}$ & $\begin{array}{l}\mathrm{RP}_{\text {ajustada }} \\
\text { (IC 95\%) }\end{array}$ & $\mathbf{p}$ \\
\hline \multicolumn{7}{|l|}{ Bloco I } \\
\hline \multicolumn{7}{|l|}{ Fatores socioeconômicos } \\
\hline Renda familiar per capita ${ }^{\dagger}$ & & & & $<0,001^{*}$ & & 0,134 \\
\hline$<0,25 \mathrm{SM}$ & 505 & $199(39,4)$ & $2,0(1,4-2,8)$ & & $1,4(1,0-2,1)$ & \\
\hline $0,25-0,5 \mathrm{SM}$ & 289 & $97(33,6)$ & $1,7(1,1-1,9)$ & & $1,4(0,9-2,0)$ & \\
\hline$\geq 0,50 \mathrm{SM}$ & 130 & $26(20,0)$ & 1,0 & & 1,0 & \\
\hline Bens de consumo & & & & $<0,001$ & & 0,083 \\
\hline 0-2 bens & 430 & $430(41,9)$ & $1,4(1,2-1,7)$ & & $1,2(1,0-1,5)$ & \\
\hline 3-5 bens & 515 & $515(29,1)$ & 1,0 & & 1,0 & \\
\hline Escolaridade materna & & & & 0,009 & & 0,756 \\
\hline Nenhuma & 82 & $29(35,4)$ & $1,4(0,9-2,0)$ & & $0,9(0,6-1,4)$ & \\
\hline $1-8$ anos & 660 & $251(38,0)$ & $1,5(1,2-1,9)$ & & $1,1(0,8-1,5)$ & \\
\hline$\geq 9$ anos & 197 & $50(25,4)$ & 1,0 & & 1,0 & \\
\hline Trabalho materno & & & & 0,174 & & 0,965 \\
\hline Sim & 210 & $65(31,0)$ & 1,0 & & 1,0 & \\
\hline Não & 733 & $265(36,2)$ & $1,2(0,9-1,5)$ & & $1,0(0,8-1,3)$ & \\
\hline \multicolumn{7}{|l|}{ Fatores ambientais } \\
\hline Estrato geográfico & & & & 0,478 & & \\
\hline RMR & 209 & $69(33,0)$ & 1,0 & & & \\
\hline IU & 226 & $74(32,7)$ & $1,0(0,8-1,3)$ & & & \\
\hline IR & 510 & $187(36,7)$ & $1,1(0,9-1,4)$ & & & \\
\hline Tipo de habitação & & & & 0,329 & & \\
\hline Alvenaria/Tijolo & 899 & $311(34,6)$ & 1,0 & & & \\
\hline Outros & 46 & $19(41,3)$ & $1,2(0,8-1,7)$ & & & \\
\hline Abastecimento de água & & & & 0,519 & & \\
\hline Rede geral & 466 & $158(33,9)$ & 1,0 & & & \\
\hline Outros & 479 & $172(35,9)$ & $1,1(0,9-1,3)$ & & & \\
\hline Tratamento de água de beber & & & & $<0,001$ & & 0,170 \\
\hline Adequado & 426 & $119(27,9)$ & 1,0 & & 1,0 & \\
\hline Não adequado & 519 & $211(40,7)$ & $1,5(1,2-1,7)$ & & $1,2(0,9-1,4)$ & \\
\hline Presença de banheiro & & & & 0,028 & & 0,513 \\
\hline Sim & 717 & $237(33,1)$ & 1,0 & & 1,0 & \\
\hline Não & 228 & $93(40,8)$ & $1,2(1-1,5)$ & & $0,9(0,8-1,1)$ & \\
\hline Destino dos dejetos & & & & $<0,001$ & & 0,104 \\
\hline Rede geral & 260 & $68(26,2)$ & 1,0 & & 1,0 & \\
\hline Outros & 683 & $260(38,1)$ & $1,5(1,2-1,8)$ & & $1,2(1,0-1,6)$ & \\
\hline Destino do lixo & & & & 0,57 & & \\
\hline Coleta pública & 513 & $175(34,1)$ & 1,0 & & & \\
\hline Outros & 432 & $155(35,9)$ & $1,1(0,9-1,3)$ & & & \\
\hline No de pessoas por cômodo & & & & $<0,001^{\star}$ & & $0,013^{*}$ \\
\hline$<1$ & 374 & $155(41,4)$ & 1,0 & & 1,0 & \\
\hline $1-2$ & 511 & $162(31,7)$ & $0,8(0,6-0,9)$ & & $0,8(0,7-1,0)$ & \\
\hline$\geq 2$ & 60 & $13(21,7)$ & $0,5(0,3-0,9)$ & & $0,6(0,4-1,1)$ & \\
\hline
\end{tabular}

continua

como fatores associados significativos o número de pessoas por cômodo, a idade materna e a idade da criança (Tabela 2). 
Tabela 2. continuação

\begin{tabular}{|c|c|c|c|c|c|c|}
\hline Blocos/Variáveis & $\begin{array}{c}\text { Amostra } \\
(\mathrm{n}=945) \\
\mathrm{n}^{\mathrm{a}}\end{array}$ & $\begin{array}{c}\text { Anemia } \\
\%\end{array}$ & $\begin{array}{c}\mathrm{RP}_{\text {bruta }} \\
\text { (IC 95\%) }\end{array}$ & $\mathrm{p}$ & $\begin{array}{l}\mathrm{RP}_{\text {ajustada }} \\
\text { (IC 95\%) }\end{array}$ & $\mathrm{p}$ \\
\hline \multicolumn{7}{|l|}{ Bloco II } \\
\hline \multicolumn{7}{|l|}{ Fatores maternos } \\
\hline Idade materna & & & & $<0,001^{*}$ & & $<0,001$ \\
\hline$<20$ anos & 98 & $57(58,2)$ & $1,9(1,5-2,4)$ & & $1,9(1,5-2,4)$ & \\
\hline 20 a-30 a & 491 & $165(33,6)$ & $1,1(0,9-1,3)$ & & $1,2(1,0-1,4)$ & \\
\hline$\geq 30 \mathrm{a}$ & 354 & $108(30,5)$ & 1,0 & & 1,0 & \\
\hline Realização de pré-natal & & & & $0,226^{*}$ & & \\
\hline Nenhuma & 35 & $15(42,9)$ & $1,3(0,8-1,9)$ & & & \\
\hline $1-5$ consultas & 243 & $89(36,6)$ & $1,1(0,9-1,3)$ & & & \\
\hline 6 ou mais & 610 & $207(33,9)$ & 1,0 & & & \\
\hline No de filhos $<5$ anos & & & & $0,009^{*}$ & & 0,811 \\
\hline 1 filho & 547 & $173(31,6)$ & 1,0 & & 1,0 & \\
\hline 2 filhos & 320 & $124(38,8)$ & $1,2(1-1,5)$ & & $1,0(0,8-1,2)$ & \\
\hline 3 ou mais filhos & 78 & $33(42,3)$ & $1,3(1-1,8)$ & & $1,0(0,8-1,4)$ & \\
\hline \multicolumn{7}{|l|}{ Acesso serviços de saúde } \\
\hline Distância serviços de saúde & & & & 0,311 & & \\
\hline $0-10 \mathrm{~min}$ & 262 & $89(34,0)$ & 1,0 & & & \\
\hline $10-30 \mathrm{~min}$ & 423 & $141(33,3)$ & $1,0(0,8-1,2)$ & & & \\
\hline$\geq 30 \mathrm{~min}$ & 247 & $96(38,9)$ & $1,1(0,9-1,4)$ & & & \\
\hline Recebe visita ACS & & & & 0,361 & & \\
\hline Sim & 840 & $289(34,4)$ & 1,0 & & & \\
\hline Não & 103 & $40(38,8)$ & $1,1(0,9-1,5)$ & & & \\
\hline \multicolumn{7}{|l|}{ Bloco III } \\
\hline \multicolumn{7}{|l|}{ Fatores relacionados à criança } \\
\hline Idade & & & & $<0,001$ & & $<0,001$ \\
\hline$<2$ anos & 326 & $195(59,8)$ & $2,7(2,3-3,3)$ & & $2,7(2,2-3,2)$ & \\
\hline$\geq 2$ anos & 619 & $135(21,8)$ & 1,0 & & 1,0 & \\
\hline Sexo & & & & 0,475 & & \\
\hline Masculino & 489 & $176(36,0)$ & $1,1(0,9-1,3)$ & & & \\
\hline Feminino & 456 & $154(33,8)$ & 1,0 & & & \\
\hline Peso ao nascer & & & & 0,591 & & \\
\hline$<2.500 \mathrm{~g}$ & 79 & $30(38,0)$ & $1.1(0,8-1,5)$ & & & \\
\hline$>2.500 \mathrm{~g}$ & 837 & $293(35,0)$ & 1,0 & & & \\
\hline Suplementação vit. A & & & & 0,380 & & \\
\hline Sim & 456 & $166(36,4)$ & 1,0 & & & \\
\hline Não & 487 & $164(33,7)$ & $0,9(0,8-1,1)$ & & & \\
\hline Ocorrência diarreia ${ }^{*}$ & & & & 0,048 & & 0,646 \\
\hline Sim & 191 & $78(40,8)$ & $1,2(1,0-1,5)$ & & $1,0(0,8-1,1)$ & \\
\hline Não & 754 & $252(33,4)$ & 1,0 & & 1,0 & \\
\hline Ocorrência tosse e febre ${ }^{*}$ & & & & 0,249 & & \\
\hline Sim & 207 & $79(38,2)$ & $1.1(0,9-1,4)$ & & & \\
\hline Não & 737 & $250(33,9)$ & 1,0 & & & \\
\hline Internação hospitalar* & & & & 0,853 & & \\
\hline Sim & 166 & $59(35,5)$ & $1(0.8-1.3)$ & & & \\
\hline Não & 779 & $271(34,8)$ & 1,0 & & & \\
\hline
\end{tabular}

${ }^{a}$ Os totais parciais de cada grupo podem diferir do total da amostra em virtude de informações ignoradas pelas entrevistadas; * Utilizado teste de Wald de tendência linear; † Salário Mínimo (SM) vigente no ano de 2006 (R \$350,00);

\#Considerou-se ocorrência de diarreia nas duas últimas semanas anteriores à entrevista para a coleta dos dados, a ocorrência de tosse e febre na semana anterior e internação hospitalar da criança nos últimos doze meses anteriores à entrevista para a coleta dos dados. 
moderado problema de saúde pública². É um resultado semelhante ao encontrado por Diniz ${ }^{27}$ há cerca de 13 anos, em menores de cinco anos no estado da Paraíba (16\%), sendo contudo bem inferior às prevalências observadas no estado de Sergipe $(22,5 \%)^{28}$ e um pouco abaixo dos valores relatados pela II Pesquisa Estadual de Saúde e Nutrição (II PESN-PE/1997) ${ }^{29}$, à época com proporções de DVA de 19,3\%.

No que concerne à população cadastrada pelas equipes da ESF, recente estudo realizado em dois municípios do semiárido mineiro no Vale do Jequitinhonha, com amostra total de 692 crianças entre 6 e 71 meses de idade, evidenciou prevalências de 11,5\% em Novo Cruzeiro e 18,2\% em Francisco Badaró ${ }^{30}$. A escassez de informações sobre este déficit nutricional em crianças cadastradas nas ESF dificultam maiores comparações, mas ao que parece, recentemente, em regiões historicamente consideradas endêmicas, as prevalências se equivalem.

Não foi detectada associação entre DVA e renda familiar per capita, apesar de valor próximo à significância $(p=0,075)$ na análise bivariada, bem como em relação à escolaridade materna, a exemplo do encontrado por Santos et al. ${ }^{31}$ em préescolares de áreas urbanas de municípios do semiárido baiano e por Pereira et al..$^{32} \mathrm{em}$ crianças de 36 a 83 meses matriculadas em uma creche da periferia da cidade de Terezina (PI). Quanto ao estrato geográfico, não houve diferenças significativas entre áreas urbanas ou rurais, apesar de se relatar maiores prevalências de níveis baixos de retinol sérico em crianças de estratos rurais ${ }^{30,33}$.

Ainda em relação aos fatores ambientais, o inadequado destino do lixo (jogado à céu aberto, enterrado, queimado) mostrou-se significativamente associado à DVA com chance quase duas vezes maior para a ocorrência do evento. É um resultado semelhante ao observado por Tei$x \operatorname{cira}^{30}$ no município de Francisco Badaró (MG) $(\mathrm{p}<0,001 ; \mathrm{OR}: 7,5)$. Crianças que são expostas a condições ambientais adversas, seja no domicílio ou peridomicílio, estão mais sujeitas a morbidades que podem comprometer ou mesmo agravar seu estado nutricional.

Neste estudo, pouco mais de $11 \%$ da amostra referiu não receber a visita do ACS, havendo pouca variação nas prevalências de DVA entre os dois grupos e ausência de associação significante. Isto contraria o observado no município de Nova Cruzeiro $(\mathrm{MG})^{30}$, onde a ausência de visitas da equipe da ESF configurou-se como importante fator de risco para este desfecho ( $\mathrm{p}=$ 0,004; OR: 4,56). Avaliar a qualidade da visita domiciliar não foi objetivo deste estudo, entretanto é pertinente considerar que a cobertura de determinada ação não garante a efetividade da mesma. Destaca-se que a visita domiciliar constitui uma boa oportunidade de aproximação da realidade das famílias e de criação de vínculos entre a equipe de saúde e a comunidade, permitindo a promoção de ações de educação em saúde, particularizando-se aqui a abordagem de temas sobre alimentação e nutrição.

Dentre os fatores relacionados à criança, ainda que as variáveis peso ao nascer e ocorrência de diarreia tenham aparecido como significantes ( $p<0,05)$, apenas a última manteve-se associada ao desfecho. O fato de que as condições de saneamento e as diarreias tenham se mantido como variáveis significativamente associadas à DVA no modelo final da análise multivariada sugere, de fato, um contexto de risco para a DVA, o que, ademais, concorda com a própria observação sobre os riscos de complicações e morte nas situações em que a DVA e as diarreias coexistem $^{34,35}$. É interessante considerar que por mecanismos não bem definidos, a vitamina A modula a resposta imunológica da mucosa intestinal, diminuindo a duração e a gravidade da doença diarreica $^{36-38}$. A recíproca é verdadeira, as diarreias tendem a agravar a DVA 2 .

A propósito de não ter sido observada associação entre suplementação anterior de vitamina A e DVA, sabe-se que esta é uma importante medida de controle, a curto prazo, dos baixos níveis de retinol sérico, especialmente em crianças que apresentam esta deficiência nutricional pela ingestão inadequada de alimentos ricos em vitamina $\mathrm{A}^{39,40}$. Ademais, alguns autores tem mostrado correlação entre estado nutricional da vitamina A e ferro, admitindo que a suplementação de vitamina A, juntamente com medidas de fortificação alimentar, poderia contribuir na redução da anemia ${ }^{41,42}$.

A prevalência de anemia (35\%) encontrada no presente estudo, a caracteriza como moderado problema de saúde pública ${ }^{1}$, sendo semelhante aos 34\% de ocorrência observada na III PESN$\mathrm{PE} / 2006^{18}$. Alguns estudos de base populacional publicados no país nos últimos dez anos ${ }^{43-47}$, com crianças na mesma faixa etária que a utilizada nesta pesquisa, mostraram valores próximos ou mais elevados os quais oscilaram entre $30,2 \%{ }^{46} \mathrm{e}$ $45,2 \%{ }^{43}$. Na realidade, existe um efeito provável de colinearidade entre os dados desta investigação e os da III PESN, uma vez que $67 \%$ das crianças participantes deste inquérito nutricional possuíam cobertura da Estratégia Saúde da Família. 
Em 2006, o estado de Pernambuco contava com 1.605 equipes da ESF implantadas ${ }^{48}$. Apesar de não corresponder ao propósito deste estudo, hipoteticamente, as prevalências de DVA e anemia seriam similares às observadas no estado em um contexto mais genérico. Deve-se ter devida cautela diante desta suposição, visto que a ESF tem sofrido modificações em virtude de políticas locais que, muitas vezes, precarizam os vínculos profissionais e descaracterizam a real proposta do modelo saúde da família.

$\mathrm{Na}$ análise bruta, as variáveis pertencentes ao nível distal referentes aos fatores socioeconômicos, como renda familiar per capita, bens de consumo e escolaridade materna, mostraram-se significantemente associadas $(\mathrm{p}<0,05)$ à anemia, porém a significação estatística não se manteve após a análise ajustada. Diferentemente do observado por Leal et al. ${ }^{48}$ em crianças de 6 a 59 meses avaliadas na III PESN. Esse fato pode ter ocorrido em virtude da superposição de variáveis de confusão no que se refere às condições de vida. Apesar da renda familiar não ter permanecido no modelo, a exemplo do encontrado por Lima et al. ${ }^{24}$ em municípios da zona da mata de Pernambuco e Oliveira et al. ${ }^{47}$, no mesmo estado, muitos autores consideram a renda como fator determinante para a ocorrência de anemia ${ }^{20-22,44,49,50}$, bem como a menor escolaridade materna ${ }^{23,44,47}$. É importante referir que a anemia muitas vezes se comporta como uma endemia pansocial, ou seja, ocorrendo sem clara distinção em diferentes categorias socioeconômicas ${ }^{1}$.

Ainda no bloco I, dentre os fatores ambientais, apenas se manteve associada ao desfecho anemia, após análise multivariada, a variável número de pessoas por cômodo $(\mathrm{p}=0,013)$. No entanto, aqui foi encontrada uma associação inesperada, desde que o maior número de pessoas $(\geq 2)$ apresentou-se como possível efeito protetor (RP: 0,6; IC: 0,4-1,1) para a ocorrência da anemia. É uma condição que difere da descrita por Vieira et al. ${ }^{46}$ em pesquisa realizada no estado de Alagoas, onde a presença de cinco ou mais pessoas no domicílio, aumentou a chance do desfecho em quase duas vezes. Admite-se, no caso do presente estudo, que o fato possa ter ocorrido em virtude do pequeno tamanho da amostra na classe $\geq 2$ pessoas/cômodo.

Ainda no rol dos fatores ambientais, bloco I, encontrou-se uniformidade nas prevalências de anemia em relação ao estrato geográfico. Elevadas proporções do evento foram observadas independente da criança residir em área urbana ou rural, evidenciando uma distribuição epidemio- lógica mais homogênea da anemia, não sendo atribuída associação significante entre essas variáveis $(\mathrm{p}=0,478)$. Vieira et $\mathrm{al}^{46}$, apesar de observarem que a frequência de anemia em menores de cinco anos era significativamente maior naqueles que residiam em municípios rurais de Alagoas, esta significância estatística não permaneceu após análise multivariada.

Entre os fatores maternos, pertencentes ao nível intermediário (bloco II), a menor idade da mãe ( $<20$ anos) configurou-se como associado ( $\mathrm{p}<0,001)$ para o baixo nível de hemoglobina da criança. Resultados semelhantes foram descritos por Silva et al. ${ }^{51}$ e Miglioli et al. ${ }^{21}$ ao analisarem a anemia no binômio mãe-filho em Pernambuco. Deve-se considerar que nesta faixa etária, a demanda de ferro é especialmente elevada, imposta pelo crescimento corporal da própria adolescência ${ }^{52}$, sobrepondo-se a maior utilização das reservas de ferro durante a gestação ${ }^{53} \mathrm{e}$ que, muitas vezes, não são repostas através de um consumo alimentar adequado.

Em relação à menor idade da criança $(<2$ anos), no grupo de fatores proximais (bloco III), esta variável permaneceu no modelo final como fortemente associada à anemia ( $\mathrm{p}<0,001)$, aumentando em quase três vezes a chance de ocorrência em relação às crianças com dois ou mais anos. O resultado é corroborado por muitos autores $^{21-23,43-47,51}$, os quais relacionam às maiores prevalências de anemia em crianças nesta faixa etária, por conta de fatores como maior velocidade de crescimento, desmame precoce, maior consumo de leite de vaca, baixa ingestão de alimentos ricos em ferro e maiores prevalências de doenças como diarreias e infecções respiratórias. A propósito, neste estudo, a ocorrência de diarreia perdeu sua significância estatística ( $\mathrm{p}<0,048)$, após afastados possíveis variáveis confundidoras, diferindo dos resultados descritos por Lima et al. ${ }^{24} \mathrm{em}$ municípios da Zona da Mata pernambucana.

Apesar do baixo peso ao nascer (BPN) não ter sido associado à anemia neste estudo, em concordância com outros autores ${ }^{21-23}$, não há um consenso nesta observação ${ }^{24}$. Sabe-se que a deficiência materna de ferro pode resultar, entre outros eventos adversos, em maior frequência de $\mathrm{BPN}^{52,54}$. Assim, uma vez que essas crianças sejam expostas a fatores externos de risco, a exemplo do ambiente desfavorável, precárias condições socioeconômicas e pouco acesso a alimentos ricos em ferro, esta circunstância poderia se prolongar ou mesmo se agravar.

A despeito das limitações metodológicas do presente estudo, sabe-se que trabalhar com ban- 
co de dados, restringe a investigação às variáveis disponíveis, impossibilitando a verificação de outros fatores concorrentes para o evento. Todavia, esta restrição não compromete o sentido e o valor de sua contribuição, ao constituir-se em um dos poucos trabalhos objetivando, a partir de dados populacionais, uma visão mais genérica do déficit nutricional de ferro e vitamina A na população assistida pela Estratégia Saúde da Família (ESF) em todo um estado brasileiro.

Com base no aqui relatado, verifica-se que, sendo um estudo de marcante pioneirismo, não se chega a todas as respostas que seriam desejáveis. Permite, no entanto, o dimensionamento inicial da magnitude da anemia e da DVA num espaço geográfico geralmente caracterizado por contrastes ambientais e sociais.

Os fatores observados como associados aos desfechos requerem uma ação intersetorial e não exclusivamente do setor saúde. Neste sentido, a prevenção destes agravos perpassa não apenas pelo apropriado acompanhamento das gestantes e crianças pertencentes aos territórios das ESF, capacitação das equipes e medidas de educação em saúde, em particular aquelas voltadas à alimentação e nutrição, mas também pela implementação de ações que permitam o incremento das condições de saneamento, habitação e socioeconômicas da população. O presente estudo pode valer como ponto de partida nesta direção.

\section{Colaboradores}

WKA Silva de Paula, MFC Caminha, JN Figueirôa e M Batista Filho participaram igualmente de todas as etapas de elaboração do artigo. 


\section{Referências}

1. World Health Organization (WHO). Worldwide prevalence of anaemia 1993-2005: WHO Global Database on Anaemia. Geneva: WHO; 2008. [homepage na Internet]. [acessado 2010 maio 19]. Disponível em: http://whqlibdoc.who.int/publications/2008/ 9789241596657_eng.pdf

2. World Health Organization (WHO). Global prevalence of vitamin A deficiency in populations at risk 1995-2005: WHO Global Database on vitamin A deficiency. Geneva: WHO; 2009. [homepage na Internet]. [acessado 2010 maio 19]. Disponível em: http:/ /whqlibdoc.who.int/publications/2009/ 9789241598019_eng.pdf

3. Brasil. Ministério da Saúde (MS). Unicef. Cadernos de Atenção Básica: Carências de Micronutrientes. In: Schmitz BAS, organizadora. Cadernos de Atenção Básica: Carências de Micronutrientes. Brasília: MS, Unicef; 2007. Brasília: MS; 2007. p. 6-22.

4. World Health Organization (WHO). Worldwide prevalence of anaemia, WHO Vitamin and Mineral Nutrition Information System, 1993-2005. Public Health Nutr 2009; 12(4):444-454.

5. Brasil. Ministério da Saúde (MS). Centro Brasileiro de Análise e Planejamento. Pesquisa Nacional de Demografia e Saúde da Criança e da Mulher - PNDS 2006: dimensões do processo reprodutivo e da saúde da criança. Brasília: MS; 2009. p. 224-253.

6. United Nation Children's Fund (Unicef). World Health Organization (WHO). Preventing iron deficiency in women and children. Thechnical Consensus on Key Issues. 1998, p. 59. In: Schmitz BAS, organizadora. Brasil. Ministério da Saúde (MS). Unicef. Cadernos de Atenção Básica: Carências de Micronutrientes. Brasília: MS, Unicef; 2007. p. 30-31.

7. Milagres RCRM, Nunes LC, Pinheiro-Sant'Ana HM. A deficiência de vitamina A em crianças no Brasil e no mundo. Cien Saude Colet 2007; 12(5):1253-1266.

8. Ramalho RA, Flores H, Saunders C. Hipovitaminose A no Brasil: um problema de saúde pública. Rev Panam Salud Publica 2002; 12(2):117-122.

9. Brasil. Ministério da Saúde (MS). Secretaria de Atenção à Saúde. Departamento de Atenção Básica. Política nacional de alimentação e nutrição. 2a ed. rev. Brasília: MS; 2003. p. 23-25. [Internet]. [acessado 2010 abr 13]. Disponível em: http://nutricao. saude.gov.br/documentos/pnan.pdf

10. Agência Nacional de Vigilância Sanitária (Anvisa). Resolução RDC no 344, de 13 de dezembro de 2002. Regulamento técnico para fortificação das farinhas de milho e trigo com ferro e ácido fólico. Diário Oficial da União 2002; $18 \mathrm{dez}$.

11. Brasil. Ministério da Saúde (MS). Portaria no 729, de 13 de maio de 2005. Institui o Programa Nacional de Suplementação de Vitamina A e dá outras providências. Diário Oficial da União 2005; 14 maio.

12. Brasil. Ministério da Saúde (MS). Secretaria de Atenção à Saúde. Departamento de Atenção Básica. Manual operacional do Programa Nacional de Suplementação de Ferro. Brasília: MS; 2005.
13. Brasil. Ministério da Saúde (MS). Secretaria de Atenção à Saúde. Departamento de Atenção Básica. Coordenação Geral da Política Nacional de Alimentação e Nutrição. Documento-base de subsídio do Seminário Estadual de Alimentação e Nutrição no SUS. Brasília: MS; 2009. 28 p. [Internet]. [acessado $2010 \mathrm{abr}$ 13]. Disponível em: http://nutricao. saude.gov.br/ docs/geral/documentobase_semi_estadual.pdf

14. Brasil. Ministério da Saúde (MS). Departamento de Atenção Básica. Atenção Básica e Saúde da Família. Diretriz conceitual. [Internet]. [acessado $2010 \mathrm{abr}$ 15]. Disponível em: http://dab.saude.gov.br/atencao basica.php

15. Rosa WAG, Labate RC. Programa saúde da família: a construção de um novo modelo de assistência. Rev. Latino-Am. Enfermagem 2005; 13(6):1027-1034.

16. Brasil. Ministério da Saúde (MS). Portaria no 648, de 28 de março de 2006. Aprova a Política Nacional de Atenção Básica, estabelecendo a revisão de diretrizes e normas para a organização da Atenção Básica para o Programa Saúde da Família (PSF) e o Programa Agentes Comunitários de Saúde (PACS). Diário Oficial da União 2006; 29 mar.

17. Brasil. Ministério da Saúde (MS). Departamento de Atenção Básica. Histórico de cobertura do PSF. Evolução da população coberta por Equipes de Saúde da Família Implantadas. [Internet]. [acessado 2011 mar 16]. Disponível em: http://dab.saude.gov.br/ abnumeros.php\#historico

18. Universidade Federal de Pernambuco. Instituto Materno Infantil Prof. Fernando Figueira. Secretaria Estadual de Saúde de Pernambuco. III Pesquisa Estadual de Saúde e Nutrição. Situação Alimentar, Nutricional e de Saúde no Estado de Pernambuco: contexto socioeconômico e de serviços. Recife: [s.n.]; 2007.

19. Furr HC, Clifford AJ, Jones AD. Analysis of apocratonenoids and retinoids by capillary gas chromatography-mass spectrometry. Methods Enzymol 1992; 213:281-90.

20. Datasus. Sistema de Informação da Atenção Básica. Cadastramento Familiar - Pernambuco. [homepage na internet]. [acessado 2010 ago 31]. Disponível em: http://tabnet.datasus.gov.br/cgi/deftohtm.exe? siab/cnv/siabfPE.def

21. Miglioli TC, Brito AM, Lira PIC, Figueroa JN, Batista Filho M. Anemia no binômio mãe-filho no Estado de Pernambuco, Brasil. Cad Saude Publica 2010; 26(9):1807-1820.

22. Silva LSM, Giuglian ERJ, Aerts DRGC. Prevalência e determinantes de anemia em crianças de Porto Alegre, RS, Brasil. Rev Saude Publica 2001; 35(1):66-73.

23. Assunção MCF, Santos IS, Barros AJD, Gigante DP, Victora CG. Anemia em menores de seis anos: estudo de base populacional em Pelotas, RS. Rev Saude Publica 2007; 41(3):328-335. 
24. Lima ACVMS, Lira PIC, Romani SAM, Eickmann SH, Piscoya MD, Lima MC. Fatores determinantes dos níveis de hemoglobina em crianças aos 12 meses de vida na Zona da Mata Meridional de Pernambuco. Rev. Bras. Saude Mater. Infant. 2004; 4(1): 35-43.

25. Victora CG, Huttly SR, Fuchs SC, Olinto MTA. The role of conceptual frameworks in epidemiological analysis: a hierarchical approach. Int J Epidemiol 1997; 26(1):224-227.

26. Brasil. Ministério da Saúde (MS). Conselho Nacional de Saúde. Resolução no. 196 de 10 de outubro de 1996. Diretrizes e Normas Regulamentadoras de Pesquisas Envolvendo Seres Humanos. Diário Oficial da União 1996; 16 out.

27. Diniz AS. Aspectos clínicos, subclínicos e epidemiológicos da hipovitaminose A em pré-escolares no estado da Paraíba [tese]. Recife: Universidade Federal de Pernambuco; 1997. In: Milagres RCRM, Nunes LC, Pinheiro-Sant'AHM. A deficiência de vitamina A em crianças no Brasil e no mundo. Cien Saude Colet 2007; 12(5):1253-1266.

28. Martins MC, Santos LMP, Assis AMO. Prevalência da hipovitaminose A em pré-escolares no Estado de Sergipe, 1998. Rev Saude Publica 2004; 38(4):537542.

29. Instituto Nacional de Alimentação e Nutrição (INAN). Instituto Materno Infantil de Pernambuco. II Pesquisa Estadual de Saúde e Nutrição: saúde, nutrição, alimentação e condições sócio-econômicas no estado de Pernambuco. Recife: INAN/ IMIP; 1998.

30. Teixeira RA. Deficiência de vitamina A e fatores associados em crianças e adolescentes em dois municípios do semi-árido de Minas Gerais [tese]. Belo Horizonte: Universidade Federal de Minas Gerais; 2010.

31. Santos LMP, Assis AMO, Martins MC, Araújo MPN, Morris SS, Barreto ML. Situação nutricional e alimentar de pré-escolares no semi-árido da Bahia (Brasil): II $\neg$ Hipovitaminose A. Rev Saude Publica 1996; 30(1):67-74

32. Pereira JA, Paiva AA, Bergamaschi DP, Rondó PHC, Oliveira GC, Lopes IBM, Illison VK, GonçalvesCarvalho CMR. Concentrações de retinol e de betacaroteno séricos e perfil nutricional de crianças em Teresina, Piauí, Brasil. Rev. bras. epidemiol. 2008; 11(2):287-296

33. Graebner IT, Saito CH, Souza EMT. Avaliação bioquímica de vitamina A em escolares de uma comunidade rural. J. Pediatr. 2007; 83(3):247-252.

34. Velasquez-Melendez G, Okani ET, Kiertsman B, Roncada MJ. Níveis plasmáticos de vitamina A, carotenóides e proteína ligadora de retinol em crianças com infecções respiratórias agudas e doenças diarréicas. Rev Saude Publica 1994; 28(5):357-364.

35. Walker CLF, Black RE. Micronutrients and Diarrhea Disease. Clin Infect Dis. 2007; 45(Supl. 1):S73S77.
36. Long KZ, Estrada-Garcia T, Rosado JL, Santos JI, Hass M, Firestone M, Bhagwat J, Young C, DuPont HL, Hertzmark E, Nanthakumar NN. The Effect of Vitamin A Supplementation on the Intestinal Immune Response in Mexican Children Is Modified by Pathogen Infections and Diarrhea. J Nutr 2006; 136(5):1365-1370.

37. Villamor E, Fawzi WW. Vitamin A Supplementation: Implications for Morbidity and Mortality in Children. J Infect Dis 2000; 182(Supl. 1):S122-S133.

38. Oliveira JM, Rondó PHC. Evidências do impacto da suplementação de vitamina A no grupo materno-infantil. Cad Saude Publica 2007; 23(11):25652575.

39. Paiva AA, Rondó PHC, Gonçalves-Carvalho CMR, Illison VK, Pereira JA, Vaz-de-Lima LRA, Oliveira CA, Ueda M, Bergamaschi DP. Prevalência de deficiência de vitamina A e fatores associados em préescolares de Teresina, Piauí, Brasil. Cad Saude Publica 2006; 22(9):1979-1987.

40. Ramalho RA, Anjos LA, Flores H. Valores séricos de vitamina A e teste terapêutico em pré-escolares atendidos em uma Unidade de Saúde do Rio de Janeiro, Brasil. Rev. Nutr. 2001; 14(1):5-12.

41. Netto MP, Priore SE, Franceschini SCC. Interação entre vitamina $\mathrm{A}$ e ferro em diferentes grupos populacionais. Rev. Bras. Saude Mater. Infant. 2007; 7(1):15-22.

42. Silva RCR, Assis AMO, Santana MLP, Barreto ML, Brito LL, Reis MG, Parraga IM, Blanton RE. Relação entre os níveis de vitamina $\mathrm{A}$ e os marcadores bioquímicos do estado nutricional de ferro em crianças e adolescentes. Rev. Nutr. 2008; 21(3):285-291.

43. Levy-Costa RB, Monteiro CA. Consumo de leite de vaca e anemia na infância no Município de São Paulo. Rev Saude Publica 2004; 38(6):797-803.

44. Oliveira MAA, Osório MM, Raposo MCF. Concentração de hemoglobina e anemia em crianças no Estado de Pernambuco, Brasil: fatores sócio-econômicos e de consumo alimentar associados. Cad Saude Publica 2006; 22(10):2169-2178.

45. Muniz PT, Castro TG, Araújo TS, Nunes NB, SilvaNunes M, Hoffmann EHE, Ferreira MU, Cardoso MA. Child health and nutrition in the Western Brazilian Amazon: population-based surveys in two counties in Acre State. Cad Saude Publica 2007; 23(6):1283-1293.

46. Vieira RCS, Ferreira HS, Costa ACS, Moura FA, Florêncio TMMT, Torres ZMC. Prevalência e fatores de risco para anemia em crianças pré-escolares do Estado de Alagoas, Brasil. Rev. Bras. Saude Mater. Infant. 2010; 10(1):107-116.

47. Oliveira MAA, Osório MM, Raposo MCF. Fatores socioeconômicos e dietéticos de risco para a anemia em crianças de 6 a 59 meses de idade. J. Pediatr. 2007; 83(1):39-46.

48. Leal LP, Batista Filho M, Lira PIC, Figueiroa JN, Osório MM. Prevalência da anemia e fatores associados em crianças de seis a 59 meses de Pernambuco. Rev Saude Publica 2011; 45(3):457-466. 
49. Oliveira APDN, Pascoal MN, Santos LC, Pereira SCL, Justino LEH, Petarli GB, Kitoko PM. Prevalência de anemia e sua associação com aspectos sociodemográficos e antropométricos em crianças de Vitória, Espírito Santo, Brasil. Cien Saude Colet 2013; 18(11):3273-3280

50. Monteiro CA, Szarfarc SC, Mondini L. Tendência secular da anemia na infância na cidade de São Paulo (1984-1996). Rev Saude Publica 2000; 34(6):6272 .

51. Silva SCL, Batista Filho M, Miglioli TC. Prevalência e fatores de risco de anemia em mães e filhos no Estado de Pernambuco. Rev. bras. epidemiol. 2008; 11(2):266-277.

52 Smith NJ, Rosello S. Iron deficiency in infancy and childhood. Am J Clin Nutr 1953; 1(4):275-286.

53. Allen LH. Anemia and iron deficiency: effects on pregnancy outcome. Am J Clin Nutr 2000; 71(5): 1280S-1284S.

54. Abu-Saad K, Fraser D. Maternal nutrition and birth outcomes. Epidemiol Rev 2010; 32(1):5-25.

Artigo apresentado em 16/03/2013

Aprovado em 27/04/2013

Versão final apresentada em 17/05/2013 